Vietnam Journal of Earth Sciences 39(2), 155-166, DOI: 10.15625/0866-7187/39/2/9702

Vietnam Academy of Science and Technology
Vietnam Journal of Earth Sciences
(VAST)

\title{
Construction of initial national quasi-geoid model VIGAC2017, first step to national spatial reference system in Vietnam
}

\author{
Ha Minh Hoa \\ Vietnam Institute of Geodesy and Cartography
}

Received 08 March 2017. Accepted 31 March 2017

\section{ABSTRACT}

Vietnam national WGS-84 reference ellipsoid was obtained in 1999 from results of an orientation of the global WGS-84 reference ellipsoid. However, usage of the broadcast satellite messages doest not give high accuracy in determination of national quasi-geoid heights. Based on the determined geopotential of the Hon Dau local geoid and constructed initial mixed quasi-geoid model VIGAC2014, this scientific article presents results of building of initial national quasi-geoid model VIGAC2017. Used data consisting of geodetic coordinates $B, L, H$ of 164 first and second orders benchmarks of the national leveling networks was obtained from GPS data processing in ITRF according to global WGS-84 ellipsoid with satellite ephemeris accuracy at level of $\pm 2,5 \mathrm{~cm}$, and the initial mixed quasi-geoid model VIGAC2014 was constructed from the EGM2008 model. The orientation of the WGS-84 ellipsoid was accomplished under conditions of it's best fitting to the Hon Dau local quasi-geoid and the parallelism of its axes to the corresponding axes of the national WGS-84 reference ellipsoid allows get national quasi-geoid heights $\zeta$ and coor-

dinate transformation parameters $d X_{0}, d Y_{0}, d Z_{0}$, that have been used for conversion of the mixed quasi-geoid heights from the VIGAC2014 quasi-geoid model to the initial national quasi-geoid model VIGAC2017.

Along withquasi-geoid heights $\zeta^{*}$, which were obtained from the initial national quasi-geoid model VIGAC2017, an estimation of the accuracy of differences $\left(\zeta-\zeta^{*}\right)$ shows that quasi-geoid heights $\zeta^{*}$ have the accuracy at the level of $\pm 6,2 \mathrm{~cm}$. Apart from that determination of seven coordinate transformation parameters $d X_{0}, d Y_{0}, d Z_{0}, \varepsilon_{X}, \varepsilon_{Y}, \varepsilon_{Z}, \Delta m$ leads to the building of the initial national spatial reference system in Vietnam.

Keywords: Global quasi-geoid, local quasi-geoid, mixed quasi-geoid, orientation of ellipsoid.

(C)2017 Vietnam Academy of Science and Technology

\section{Introduction}

In history of construction of national vertical reference systems in the world, starting from point of view of German mathematician

"Corresponding author, Email: minhhoavigac@gmail.com
Carl Friedrich Gauss (1777 - 1855) in 1828

(Gauss C.F., 1828) about a coincidence of the geoid with an undisturbed mean sea level on the oceans and proposal of German mathematician Johann Benedict Listing (1808 - 1882) in 1873 (Listing J.B., 1873) about the usage of the geoid for initial surface of the vertical ref- 
erence systems, every country or group of different countries used a mean sea level at the zero tide gauge station. In Vietnam, tide gauge station of Hon Dau is used for the construction of national or regional vertical reference system. At present, we know that the geoid didn't coincide with the mean sea level on oceans, geopotential $\bar{W}_{0}=62636856,0 \mathrm{~m}^{2} \cdot \mathrm{s}^{-2}$ on the surface of the global geoid had been determined by altimetry data (Bursa M., Kenyon S, et al., 2007)and accepted by IERS (Petit G., Luzum B., 2010). Abovementioned achievement gives ability to determine the geopotential $W_{0}$ of the local geoid, best fitting to mean sea lev$\mathrm{el}$ at zero tide gauge station. In Vietnam, geopotential $W_{0}=62636847,2911 \mathrm{~m}^{2} \cdot \mathrm{s}^{-2}$ of the Hon Dau local geoid was announced in (Ha Minh Hoa et al., 2012; Ha Minh Hoa, 2013b; Ha Minh Hoa, 2014b). Because the Hon Dau local quasi-geoid coincides with the Hon Dau local geoid on the sea and it has been used for the initial surface of vertical reference system of Hai Phong 1972 (HP72), the usage of the Hon Dau local quasi-geoid for solving the task of ellipsoid orientation creates important base of construction of the high accurate national quasi-geoid model.

In Vietnam GNSS technology is widely used for research of the Earth crustal movement or ionosphere disturbances during the magnetic storm (Le Huy Minh et al., 2016; Vy Quoc Hai et al., 2016, Ha Minh Hoa, Dang Hung Vo et al., 2005) proposed the construction of the national dynamic coordinate system, that in fact is national spatial reference system with the purpose of closely connecting to ITRF. In addition, the construction of the national spatial reference system is the most important content of Development Strategy of Geodesy and Cartography to the 2020 year by Decision No. 33/2008/QĐ-TTg of the prime minister on 27 February 2008.
Thanks to GNSS technology, we get high accurate geodetic coordinates B, L in VN2000 2D. However, getting geodetic height $\mathrm{H}$ requires the high accurate national quasi-geoid model. (Ha Minh Hoa et al., 2012; Ha Minh Hoa, 2014a) analyzed scientific base for the construction of the national dynamic coordinate system, in which the most important task is a creation of the high accurate national quasi-geoid model with accuracy more than \pm 4 $\mathrm{cm}$ to get spatial coordinates of geodetic points with relative accuracy at level $10^{-9}$ by international regulation. For that, we must return to solve the task of the orientation of global WGS-84 ellipsoid best fitting to the Hon Dau local quasi-geoid.

Solving above-mentioned task, we will get coordinate transformation $d X_{0}, d Y_{0}, d Z_{0}$, which are spatial coordinates of the center of the WGS-84 global reference ellipsoid according to the center of the WGS-84 national (local) reference ellipsoid. Hence we will obtain two types of data:

- Data of type 1: Geodetic coordinates $B, L, H$ of GNSS points, with being used for solving the task of the orientation of ellipsoid in the national spatial reference system VN2000 - 3D. Global WGS-84 reference ellipsoid oriented under the condition of the best fitting to the Hon Dau local quasi-geoid will become the WGS-84 national (local) reference ellipsoid (Figure 1);

- Data of type 2: National quasi-geoid heights $\zeta$ of GNSS points.

For the purpose of construction of the high accurate national quasi-geoid model, we are only interested in data of type 2 . Thus, the high accurate national quasi-geoid model is the model of quasi-geoid heights $\zeta$ of specific points on the surface of the Hon Dau local quasi-geoid according to the surface of the WGS-84 national reference ellipsoid.

For solving the task of orientation of ellipsoid, we must create a GNSS network on whole territory of Vietnam and accomplish 
processing of GNSS data in ITRF on base of the using of satellite ephemeris with accuracy at the level $\pm 2,5 \mathrm{~cm}$, which allows getting global geodetic $\bar{H}$ (Figure 1) with accuracy at the level $\pm 1,4 \mathrm{~cm}$. After processing of GNSS data in ITRF, we obtain spatial coordinates $\bar{X}, \bar{Y}, \bar{Z}$ and global geodetic coordinates $\bar{B}, \bar{L}, \bar{H}$ of GNSS points according to the WGS-84 global reference ellipsoid. Apart from that, GNSS points have national normal heights $H^{\gamma}$ obtained by first and second orders differential leveling from first and second orders national benchmarks and determined from the surface of the Hon Dau local quasigeoid (Figure 1). Aforementioned GNSS points have been called as orientation points.

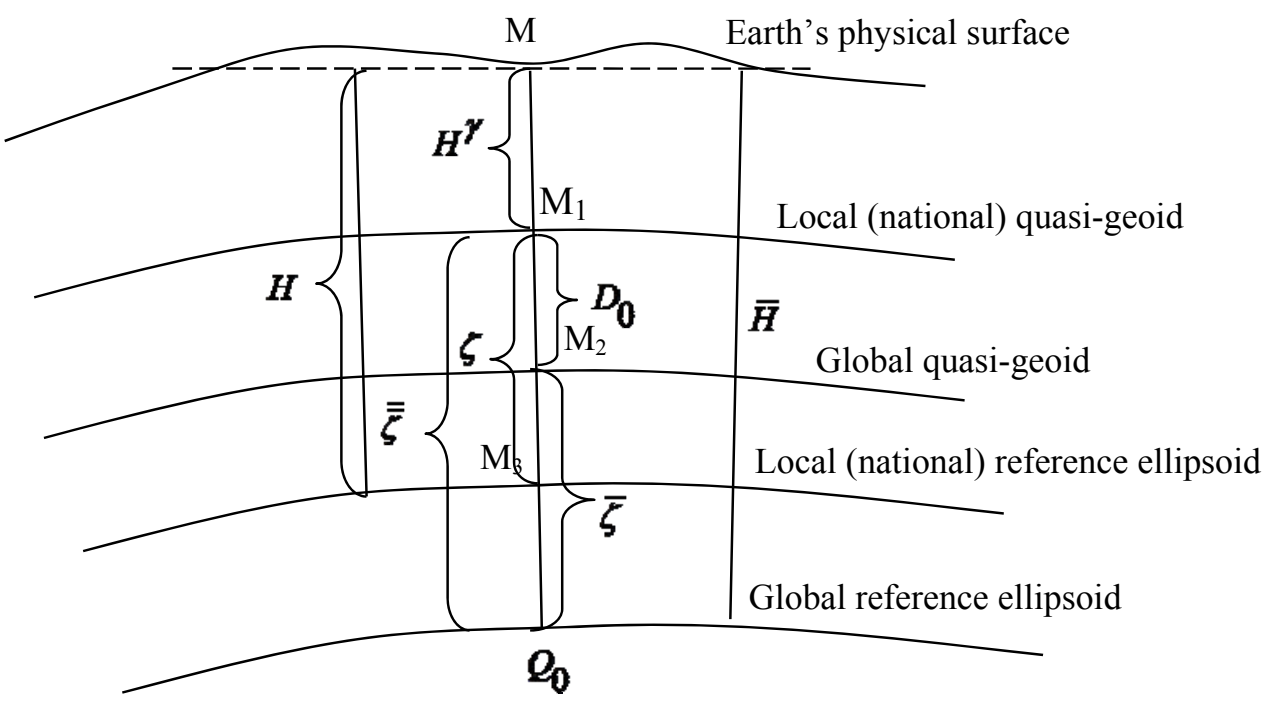

Figure 1. Relationships between local quasi-geoid, global quasi-geoid, local ellipsoid and global ellipsoid

The national quasi-geoid model is the model of heights of points $\mathrm{M}_{1}$ on the surface of the Hon Dau local quasi-geoid according to the surface of the WGS-84 national reference ellipsoid, in addition, points $\mathrm{M}_{1}$ corresponds to points $\mathrm{M}$ on the Earth's physical (Figure 1).

In Figure 1, we symbolize $\zeta$ as local quasigeoid height (national quasi-geoid height) of point $\mathrm{M}$ and is equal to segment $\mathrm{M}_{1} \mathrm{M}_{3}, \overline{\bar{\zeta}}$ as mixed quasi-geoid height of point $\mathrm{M}$ and is equal to segment $\mathrm{M}_{1} \mathrm{Q}_{0}, \bar{\zeta}$ as global quasigeoid height of point $\mathrm{M}$ and is equal to segment $\mathrm{M}_{2} \mathrm{Q}_{0}, D_{0}=M_{1} M_{2}$ as height of point $\mathrm{M}_{1}$ on the Hon Dau national (local) quasigeoid according to the global quasi-geoid.
Result of the orientation of the global ellipsoid under the condition of best it's fitting to the Hon Dau national quasi-geoid allows obtaining the national quasi-geoid height (local quasi-geoid height) $\zeta$ and the local geodetic height $H$ of GNSS point so that $H=H^{\gamma}+\zeta$. High accurate national quasigeoid heights $\zeta$ of GNSS points are very precious data for serving the construction of the high accurate national quasi-geoid model and the determination of the 07 coordinate transformation parameters from ITRF to national spatial reference system VN2000 - 3D by formula of Bursa - Wolf with the purpose of a close connection between two those spatial reference systems. The results of solving tasks of the orientation of the WGS- 84 global refer- 
ence ellipsoid under the condition of the best it's fitting to the Hon Dau local quasi-geoid, the construction of the high accurate national quasi-geoid model and the determination of the 07 coordinate transformation parameters from ITRF to national spatial reference system VN2000 - 3D by formula of Bursa - Wolf will be presented in this scientific article.

It is necessary to underline that it was seen in 1999 the accomplished orientation of the WGS - 84 global reference ellipsoid under the condition of the best fitting to the Hon Dau local quasi-geoid in the proves of the construction of the plane coordinate reference system VN2000-2D based on the GPS data of the 25 GPS points. However, in that period, the GPS data has not been processed in ITRF with the using of satellite ephemeris with accuracy at level $\pm 2,5 \mathrm{~cm}$ by software Bernese, rather being processed in WGS-84 with the usage of broadcast satellite message by software GPSuvey. Because global geodetic coordinates $\bar{B}, \bar{L}, \bar{H}$ of GPS points did not achieve high accuracy and national quasigeoid heights with the accuracy only at level $\pm 1,6 \mathrm{~m}$ (Scientific report, p.125). This accuracy satisfied requirement of reduction of measurements to ellipsoid for adjustment of the national astro - geodetic network, but did not meet the requirement of the construction of the high accurate national quasi-geoid model.

In order to construct the high accurate national quasi-geoid model, we must solve 03 problems:

Problem 1. Based on $n$ orientation points, accomplishing the orientation of the WGS-84 global reference ellipsoid under the condition of the best it's fitting to the Hon Dau local quasi-geoid, we will get 03 coordinate transformation parameters $d X_{0}, d Y_{0}, d Z_{0}$ from ITRF according to the WGS- 84 global reference ellipsoid to VN2000 - 3D according to the WGS084 national reference ellipsoid and national quasi-geoid heights $\zeta$ of the $n$ abovementioned points of orientation. This problem will be solved in 3.1.

Problem 2. Creation of relationship between the mixed quasi-geoid model and the national quasi-geoid model with the purpose of propagation of the national quasi-geoid model for the whole territory of Vietnam; Construction of the national quasi-geoid model VIGAC2017 and estimation of the accuracy of this model. This problem will be solved in 3.2 .

Problem 3. Estimation of differential rotations $\varepsilon_{X}, \varepsilon_{Y}, \varepsilon_{Z}$ and differential scale change $\Delta m$ between ITRF and VN2000 - 3D based on geodetic coordinates $\mathrm{B}, \mathrm{L}$ in VN2000 2D, national normal heights $H^{\gamma}$, global geodetic coordinates $\bar{B}, \bar{L}, \bar{H}$ of orientation points and results of solution of problem 2. This problem will be solved in 3.3.

\section{Data}

In order to solve the above-mentioned problems, we can have set of orientation points covering the whole territory of Vietnam. Accomplishing project "Construction of local geoid model on territory of Vietnam" in period 2009 - 2010 Vietnam Department of Surveying and Cartography carried out GPS observations on 290 first order benchmarks, 199 second order benchmarks and GPS data processing in ITRF by software Bernese on base of the using of satellite ephemeris with accuracy at level $\pm 2,5 \mathrm{~cm}$. Because of the displacement of some first and second orders benchmarks from social - economic activities and Earth's crustal movements, on base of Smirnov's statistic criterion selected the 89 most stable first order benchmarks and the 75 most stable second order benchmarks (Ha Minh Hoa et al., 2016a; Luong Thanh Thach, 2016). Thus, we have all 164 first, second orders benchmarks, covering over the whole territory of Vietnam, with high accurate global geodetic coordinates 
$\bar{B}, \bar{L}, \bar{H}$ according to the WGS-84 global reference ellipsoid, and use them as orientation points for solving abovementioned problems. Ha Minh Hoa, et al., (2012); Ha Minh Hoa, (2013b); Ha Minh Hoa et al., (2016a) determined geopotential $W_{0}=62636847,2911 \mathrm{~m}^{2} \cdot \mathrm{s}^{-2}$ of the Hon Dau local geoid and height $D_{0}=0,890 \mathrm{~m}$ of the Hon Dau local quasi-geoid according to the global quasi-geoid. Estimation of height $D_{0}$ shows that it is constant on whole territory of Vietnam (Ha Minh Hoa, et al., 2012; Nguyen Tuan Anh, 2015) and in global scale (Ha Minh Hoa, 2016b). With above-presented research results, we can calculate mixed quasigeoid height $\overline{\bar{\zeta}}^{*}$ from global quasi-geoid height $\bar{\zeta}$ by the following formula:

$$
\overline{\bar{\zeta}}^{*}=\bar{\zeta}+D_{0}=\bar{\zeta}+0,890 \mathrm{~m}
$$

where $\bar{\zeta}$ is the global quasi-geoid height determined from the EGM2008.

Formula (1) has been used for the construction of the mixed quasi-geoid model VIGAC2014 in the state order science technological theme (Ha Minh Hoa et al., 2016a). The accuracy of mixed quasi-geoid model VIGAC2014 has obtained at level \pm 7 $\mathrm{cm}$ based on the 89 first-order benchmarks (Ha Minh Hoa et al., 2016a) and at level $\pm 8 \mathrm{~cm}$ based on the 75 second order benchmarks (Luong Thanh Thach, 2016). Above-mentioned levels of accuracy fully correspond to levels of accuracy of the first and second orders national normal heights (Ha Minh Hoa, 2014b). However, those levels of accuracy do not satisfy the requirement of accuracy more than $\pm 4 \mathrm{~cm}$ of the national quasi-geoid model used for the construction of the national spatial reference. Apart from that, the mixed quasi-geoid model VIGAC2014 is not the national quasi-geoid model. That is why we must solve problem of orientation of the WGS-84 global reference ellipsoid, best fitting to the Hon Dau local quasi-geoid, with purposes of transformation of the mixed quasi-geoid model VIGAC2014 to the national quasi-geoid model and it's accuracy estimation.

With the purpose of calculation of national normal heights by the mixed quasi-geoid model VIGAC2014 and GNSS technology, (Ha Minh Hoa, 2014b) constructed criterion for base points of mixed quasi-geoid model VIGAC2014. The result determined 09 base points such as I(HN-VL)6-1, I(HNVL)28-1，I(HN-VL)64，I(HN-VL)72, I(VLHT)98, I(VL-HT)158, I(BH-HN)33, I(BHTH)65, I(BH-TH)122A. Those base points have been the accomplished transmission of national normal heights to 30 GNSS points of the North Vietnam geodynamic network, the Cuu Long delta geodynamic network and 02 GNSS points on islands Con Dao, Phu Quoc with the maximal distance of transmission at the level of 1,500 km. On every GNSS point deviation from 09 obtained normal heights does not exceed 1,5 cm (Ha Minh Hoa et al., 2016a). This shows that differences of mixed quasi-geoid heights between arbitrary two points from the mixed quasi-geoid model VIGAC2014 have very high accuracy. So the mixed quasi-geoid model VIGAC2014 is very important data resource for the construction of the high accurate national quasi-geoid model.

\section{Applied methods}

By IAG resolution No.16 (June 1983) in Hamburg (Germany) (International Association of Geodesy (IAG), 1984), all geodetic data must be processed in the zero tide system. (Ha Minh Hoa, 2014b) presented formulas for conversion of normal height $H^{\gamma}$ from the mean tide system to the zero tide system, of global geodetic height $\bar{H}$ and global quasi-geoidheight $\bar{\zeta}$ from the free tide system to the zero tide system. In the next research of this article we understand that all normal heights, geodetic heights and quasigeoidheights belonged to the zero tide system. 


\subsection{Method of orientation of WGS-84 ellipsoid for it's best fitting to the Hon Dau local quasi-geoid}

It is assumed that we have set of $n$ orientation points. By regulation of IERS, national reference ellipsoid must be oriented so that its axes are parallel to corresponding international axes. Because the main axes of the WGS-84 global reference ellipsoid are parallel to corresponding international axes, we must orient the WGS- 84 global reference ellipsoid under the condition of the best fitting to the Hon Dau local quasi-geoid so that the axes of the WGS-84 national reference ellipsoid are parallel to the corresponding axes of the WGS-84 global reference ellipsoid.

Then for $i$-th orientation point $(i=1,2, \ldots$, n) relationship between the local geodetic height $H_{i}$ according to the WGS-84 national reference ellipsoid and the local geodetic height $\bar{H}_{i}$ according to the WGS-84 global reference ellipsoid is presented in the following form:

(Ha Minh Hoa, 2013a):

$$
H_{i}=\bar{H}_{i}+A_{i} \cdot\left(\begin{array}{c}
d X_{0} \\
d Y_{0} \\
d Z_{0}
\end{array}\right),
$$

where coefficient matrix A has form:

$A_{i}=\left(\cos \bar{B}_{i} \cdot \cos \bar{L}_{i} \quad \cos \bar{B}_{i} \cdot \sin \bar{L}_{i} \quad \sin \bar{B}_{i}\right)$, $\bar{B}_{i}, \bar{L}_{i}, \bar{H}_{i}$ are global geodetic coordinates of i-th point according to the WGS-84 global reference ellipsoid. Symbolizing $H_{i}^{\gamma}$ as national normal height of i-th orientation point, on account of $\zeta_{i}=H_{i}-H_{i}^{\gamma}$, $\overline{\bar{\zeta}}_{i}=\bar{H}_{i}-H_{i}^{\gamma}$, where $\overline{\bar{\zeta}}_{i}$ is the mixed quasigeoidheight of $i$-th point, from (2) we have the relation:

$$
\zeta_{i}=\overline{\bar{\zeta}}_{i}+A_{i} \cdot\left(\begin{array}{l}
d X_{0} \\
d Y_{0} \\
d Z_{0}
\end{array}\right)
$$

From (3) we get observation equation in following form:

$$
\zeta_{i}=A_{i} \cdot\left(\begin{array}{l}
d X_{0} \\
d Y_{0} \\
d Z_{0}
\end{array}\right)+l_{\zeta_{i}},
$$

Where constant term $l_{\zeta_{i}}=\overline{\bar{\zeta}}_{i}$.

Solving system of observation equations (4) under the condition of the best fitting of the WGS-84 global reference ellipsoid to the Hon Dau local quasi-geoid, i.e. under the condition $\sum_{i=1}^{n} \zeta_{i}^{2}=$ min, we will get coordinate transformation parameters $d X_{0}, d Y_{0}, d Z_{0}$.

From (4) we will obtain the national (local) quasi-geoid heights $\zeta$ of the $n$ orientation points. The estimation of the accuracy of the national (local) quasi-geoid heights $\zeta$ will be considered in 3.2 .

\subsection{Determination of relationship between mixed quasi-geoid model VIGAC2014 and national quasi-geoid model VIGAC2017}

As above presented, model VIGAC2014 is only the mixed quasi-geoidmodel, but is not the nationalquasi-geoidmodel. With national normal height $H^{\gamma}$ of geodetic point, mixed quasi-geoid height $\overline{\bar{\zeta}}^{*}(1)$ from the VIGAC2014 is calculated by formula $\overline{\bar{\zeta}}^{*}=\bar{H}-H^{\gamma}$, where $\bar{H}$ is the global geodetic height according to the WGS84 global reference ellipsoid, meanwhile, nationalquasi-geoidheight $\zeta$ is calculated by

formula $\zeta=H-H^{\gamma}$, where $H$ is the local geodetic height according to the WGS84 national reference ellipsoid. Model 
VIGAC2014 can be used for calculation of the national normal height $H^{\gamma}$ based on global geodetic height $\bar{H}$ obtained from GNSS technology, but can not be used for determination of local geodetic height $H$ by formula $H=H^{\gamma}+\zeta$.

In order to construct the national quasigeoid model from the mixed quasi-geoid model VIGAC2014, taking account of formula (3), we get the formula of conversion of the mixed quasi-geoid height $\overline{\bar{\zeta}}^{*}$ to the national quasi-geoid height $\zeta^{*}$ in the following form:

$$
\zeta_{i}^{*}=\bar{\zeta}_{i}^{*}+A_{i} \cdot\left(\begin{array}{l}
d X_{0} \\
d Y_{0} \\
d Z_{0}
\end{array}\right)+C,
$$

where coordinate transformation parameters $d X_{0}, d Y_{0}, d Z_{0}$ have been determined in 3.1, $\quad \mathrm{C}$ is correction from existence of systenatic error in the VIGAC2014 model.

The mixed quasi-geoid model VIGAC2014 is used for the construction of the national quasi-geoid model VIGAC2017 by formula (5) in taking account of two it's outstanding advantages:

- The mixed quasi-geoid model VIGAC2014created from the EGM2008 model allows getting difference of quasigeoid heights between two arbitrary points with very high accuracy.

- The mixed quasi-geoid model VIGAC2014 allows propagating quasi-geoid heights to big distances on the whole territory of Vietnam, even to territories of neighbor countries.

With two independent series: series of national quasi-geoid heights $\zeta$ obtained from the results of ellipsoid orientation in 3.1 and series national quasi-geoid heights $\zeta^{*}$ achieved by formula (5) from the
VIGAC2014 model, based on method of double observation processing we will accomplish the accuracy estimation of the national quasi-geoid model VIGAC2017 and determine correction $\mathrm{C}$ in formula (5).

\subsection{Determination of differential rotations} $\varepsilon_{X}, \varepsilon_{Y}, \varepsilon_{Z}$ and differential scale change $\Delta m$

Although WGS84 national reference ellipsoid has axes, paralleling to corresponding axes of the WGS-84 global reference ellipsoid, but between ITRF and VN2000 - 3D exist differential rotations $\varepsilon_{X}, \varepsilon_{Y}, \varepsilon_{Z}$ and differential scale change $\Delta m$, with being arise from error accumulation and propagation in process of approximate calculation of coordinates of the national first and second orders astro - geodetic points in

VN2000 - 2D. Values $\varepsilon_{X}, \varepsilon_{Y}, \varepsilon_{Z}, \Delta m$ with parameters $d X_{0}, d Y_{0}, d Z_{0}$, obtained in 3.1, creating 07 coordinate transformation parameters in Bursa - Wolf's formula in the following form:

$$
\left(\begin{array}{l}
X \\
Y \\
Z
\end{array}\right)=\left(\begin{array}{l}
\bar{X} \\
\bar{Y} \\
\bar{Z}
\end{array}\right)+\left(\begin{array}{l}
d X_{0} \\
d X_{0} \\
d Z_{0}
\end{array}\right)+\left[\begin{array}{ccc}
\Delta m & \varepsilon_{Z} & -\varepsilon_{Y} \\
-\varepsilon_{Z} & \Delta m & \varepsilon_{X} \\
\varepsilon_{Y} & -\varepsilon_{X} & \Delta m
\end{array}\right]\left(\begin{array}{l}
\bar{X} \\
\bar{Y} \\
\bar{Z}
\end{array}\right),
$$

where $\bar{X}, \bar{Y}, \bar{Z} \quad$ are global geodetic coordinates of geodetic points according to the WGS-84 global reference ellipsoid, $X, Y, Z$ are national (local) geodetic coordinates of this geodetic point according to the WGS-84 national reference ellipsoid.

In case of spatial coordinates $\bar{X}, \bar{Y}, \bar{Z}$ of geodetic point are known in ITRF, but national spatial coordinates $X, Y, Z$ of this geodetic point in VN2000 - 3D are calculated by formula: 


$$
\begin{aligned}
& X=(N+H) \cdot \cos B \cdot \cos L, \\
& Y=(N+H) \cdot \cos B \cdot \sin L, \\
& Z=\left[N \cdot\left(1-e^{2}\right)+H\right] \cdot \sin B .
\end{aligned}
$$

where $B, L$ are geodetic coordinates of geodetic point in VN2000 - 3D; the prime vertical radius of curvature $N$ of this point is calculated by formula:

$$
N=\frac{a}{\sqrt{1-e^{2} \cdot \sin ^{2} B}} ; \text { national geodetic }
$$

height $H=H^{\gamma}+\zeta^{*}$ with national quasi-geoid height $\zeta^{*}$, determined by formula (5).

With known coordinate transformation parameters $d X_{0}, d Y_{0}, d Z_{0}$ in 3.1, from (6) we have observation equations:

$$
\begin{aligned}
& v_{X}=-\bar{Z} \cdot \varepsilon_{Y}+\bar{Y} \cdot \varepsilon_{Z}+\bar{X} \cdot \Delta m+l_{X}, \\
& v_{Y}=\bar{Z} \cdot \varepsilon_{X}-\bar{X} \cdot \varepsilon_{Z}+\bar{Y} \cdot \Delta m+l_{Y}, \\
& v_{Z}=-\bar{Y} \cdot \varepsilon_{X}+\bar{X} \cdot \varepsilon_{Y}+\bar{Z} \cdot \Delta m+l_{Z},
\end{aligned}
$$

where constant terms $l_{X}=\bar{X}+d X_{0}-X$, $l_{Y}=\bar{Y}+d Y_{0}-Y, l_{Z}=\bar{Z}+d Z_{0}-Z$.

$$
d X_{0}=204,511083 \mathrm{~m}, d Y_{0}=42,192468
$$

national quasi-geoid heights $\zeta$ (4) of the 164 orientation points.

Minimal national quasi-geoid height $0,042 \mathrm{~m}$ belongs to the second order benchmark II(PLK - PL)24 and maximal national quasi-geoid height $4,524 \mathrm{~m}$ belongs to the first order benchmark $\mathrm{I}(\mathrm{BH}-\mathrm{TH}) 59$. Accomplishing estimation of two independent series $\zeta$ nad $\zeta^{*}$ on the 164 orientation points by method of double observation processing, we had got correction $\mathrm{C}=-0,023 \mathrm{~m}$. Differences $d_{i}=\zeta_{i}-\zeta_{i}^{*}, \mathrm{i}=1,2, \ldots, 164$, have been presented in Table 1. RMS of every from two abovementiond series is equal to $m_{\zeta}= \pm \sqrt{\frac{\sum_{i=1}^{164} d_{i}^{2}}{2 \times 164}}= \pm \sqrt{\frac{1,265}{328}}= \pm 0,062 \mathrm{~m}$.
Based on the set of orientation points, we wiil solve system of observation equations in form (7) under condition $\Sigma\left(v_{X}^{2}+v_{Y}^{2}+v_{Z}^{2}\right)=$ min and wiil get unknown parameters $\varepsilon_{X}, \varepsilon_{Y}, \varepsilon_{Z}$ and $\Delta m$.

By such way we will obtain the 07 coordinate transformation parameters $d X_{0}, d Y_{0}, d Z_{0}, \varepsilon_{X}, \varepsilon_{Y}, \varepsilon_{Z}, \Delta m$ for conversion of coordinates from ITRF according the WGS-84 global reference ellipsoid to VN2000 - 3D according the WGS-84 national reference ellipsoid.

\section{Results}

Based on global geodetic coordinates $\bar{B}_{i}, \bar{L}_{i}, \bar{H}_{i}$ on $\mathrm{n}=164$ orientation points (i $=1,2, \ldots, 164)$ we solved system of observation equations (4) under the condition $\sum_{i=1}^{164} \zeta_{i}^{2}=$ min and had the following coordinate transformation parameters:

$8 \mathrm{~m}, d Z_{0}=111,417880 \mathrm{~m}$,

Limited maximal absolute value of defferences $d$ has been determined by formula $|d|_{\max }=t \cdot \sqrt{2} \cdot m_{\zeta}$. With $\mathrm{t}=2,0$; $m_{\zeta}=0,062 \mathrm{~m}$, value $|d|_{\max }=0,175$. In Table 1 , number of absolute values of differenses $d$ in interval $(0-17,5 \mathrm{~cm})$ is160 (97,56\%). With $\mathrm{t}=2,5 ; m_{\zeta}=0,062 \mathrm{~m}$, limited maximal absolute value $|d|_{\max }=0,219$. Mean while, number of differences $d$ with absolute values in the interval $(17,6-19,5 \mathrm{~cm})$ is only 4 $(2,46 \%)$. Hence, differences $d$ in Table 1 satisfy limited value, in addition differences $d$ with small absolute values occupy vast majority. That attestes reliability of the initial national quasi-geoid model VIGAC2017, with being constructed from the mixes quasigeoidVIGAC2014 by formula (5). 
Vietnam Journal of Earth Sciences 39(2), 155-166

Based on the 164 orientation points with those geodetic corrdinates B, L in VN2000, we solved the system of observation equations in form (7) and had unknown parameters

$\varepsilon_{X}, \varepsilon_{Y}, \varepsilon_{Z}$ and $\Delta m$ with following values:

Ex $<$ second $>=-0 ", 011168229$ or Ex $<$ radian $>=-0,000000054$

Ey $<$ second $>=0$ ",085600577 or Ey $<$ radian $>=0,000000415$

$$
\begin{aligned}
& \text { Ez }<\text { second }>=-0 ", 400462723 \text { or } \mathrm{Ez} \\
& <\text { radian }>=-0,000001941 \\
& \mathrm{Dm}=0,000000000
\end{aligned}
$$

Abovepresented parameters $\varepsilon_{X}, \varepsilon_{Y}, \varepsilon_{Z}, \Delta m$ with parameters $d X_{0}, d Y_{0}, d Z_{0}$ (8) created set of the 07 coordinate transformation parameters from ITRF to VN2000 - 3D and guarantee close connection between those

\begin{tabular}{|c|c|c|c|c|c|}
\hline No & Points & $\begin{array}{c}\text { Differences } \\
\mathrm{d}(\mathrm{m})\end{array}$ & No & Points & $\begin{array}{c}\text { Differences } \\
\mathrm{d}(\mathrm{m})\end{array}$ \\
\hline \multicolumn{6}{|c|}{ Differences with absolute values not more $17.5 \mathrm{~cm}$} \\
\hline 1 & IBH-TH $122 \mathrm{~A}$ & 0.029 & 50 & IVL-HT158 & 0.023 \\
\hline 2 & IBH-TH119 & 0.049 & 51 & IDN-BT74 & 0.045 \\
\hline 3 & IBH-HN33 & 0.032 & 52 & $\mathrm{IBH}=-\mathrm{LS} 88-1$ & 0.047 \\
\hline 4 & IBH-HN39 & 0.037 & 53 & IVL-HT98 & 0.032 \\
\hline 5 & IBH-HN42 & 0.009 & 54 & IBH-LS.85-1 & 0.051 \\
\hline 6 & IHN-VL4-1 & 0.046 & 55 & IBH-LS93 & 0.049 \\
\hline 7 & IHN-VL6-1 & 0.017 & 56 & IBH-LS71 & 0.054 \\
\hline 8 & IVL-HT152-1 & -0.023 & 57 & IBT-APD56 & 0.034 \\
\hline 9 & IHN-VL34- & -0.049 & 58 & IVL-HT87 & 0.051 \\
\hline 10 & IHP-MC48A & -0.045 & 59 & IVL-HT247A & 0.045 \\
\hline 11 & IBH-TH3-1 & -0.021 & 60 & ILS-TY1 & 0.065 \\
\hline 12 & IVL-HT181 & -0.061 & 61 & IDN-BT83 & 0.052 \\
\hline 13 & ILS-TY4 & -0.037 & 62 & IVL-HT78 & 0.055 \\
\hline 14 & IVL-HT309A & -0.058 & 63 & ILS-HN36 & 0.065 \\
\hline 15 & IVL-HT317 & -0.053 & 64 & ILS-HN29 & -0.022 \\
\hline 16 & IVL-HT187 & -0.049 & 65 & IHN-VL28-1 & 0.032 \\
\hline 17 & IVL-HT170-1 & -0.048 & 66 & IIDK-TM41 & 0.021 \\
\hline 18 & IHP-MC41 & -0.019 & 67 & IIBH-XL11-1 & -0.045 \\
\hline 19 & IHN-VL56 & 0.051 & 68 & IIBH-XL17 & 0.003 \\
\hline 20 & IBH-TH1 1 & 0.064 & 69 & IIBS-CD12 & -0.047 \\
\hline 21 & IHN-VL40-1 & 0.057 & 70 & IIBS-CD3 & 0.001 \\
\hline 22 & IVL-HT130 & -0.035 & 71 & IICD-VC4-1 & -0.020 \\
\hline 23 & IBH-TH5 & -0.015 & 72 & IICT-GD10 & 0.001 \\
\hline 24 & IHN-VL38-1 & -0.019 & 73 & IICT-GD15-1 & -0.036 \\
\hline 25 & IVL-HT197 & -0.032 & 74 & IICF-VT1 & -0.039 \\
\hline 26 & IBT-APD63 & -0.032 & 75 & IIGD-AB12 & -0.057 \\
\hline 27 & IVL-HT127-3 & -0.026 & 76 & IIGD-AB9-1 & -0.036 \\
\hline 28 & IBT-APD59-1 & -0.029 & 77 & IIGD-APD6-1 & -0.036 \\
\hline 29 & IVL-HT278-1 & -0.023 & 78 & IIHN-AB11 & -0.064 \\
\hline 30 & IVL-HT108 & -0.015 & 79 & IIHN-AB3 & -0.062 \\
\hline 31 & IDN-BT77 & -0.012 & 80 & IIHN-MT5 & -0.019 \\
\hline 32 & IBT-NH17-1 & -0.015 & 81 & IILC-TG19A & -0.020 \\
\hline 33 & IVL-HT83 & -0.009 & 82 & IIMC-XM7-1 & -0.056 \\
\hline 34 & IBH-HN17 & 0.006 & 83 & IIMT-TH4 & -0.026 \\
\hline 35 & IHN-VL45-1 & 0.053 & 84 & $\mathrm{IINB}=\mathrm{HN} 15$ & 0.060 \\
\hline 36 & IBH-TH65 & 0.015 & 85 & IIPLK-PL12 & -0.034 \\
\hline 37 & IVL-HT178 & 0.001 & 86 & IIPLK-PL2 & 0.061 \\
\hline 38 & IVL-HT103 & 0.008 & 87 & IIPLK-PL8 & -0.037 \\
\hline 39 & IHN-VL64 & 0.017 & 88 & IISC-VT3-1 & -0.040 \\
\hline 40 & IVL-HT141- & 0.009 & 89 & IITX-TL25 & -0.050 \\
\hline
\end{tabular}
spatial reference systems.

Table 1. Estimation of the differences $d=\zeta-\zeta^{*}$ on the 164 first and second order benchmarks 
Ha Minh Hoa/Vietnam Journal of Earth Sciences 39 (2017)

\begin{tabular}{|c|c|c|c|c|c|}
\hline 41 & IVL-HT329A & 0.009 & 90 & IITX-TL6 & -0.048 \\
\hline 42 & IHN-VL72 & 0.024 & 91 & IIYB-CN18 & -0.055 \\
\hline 43 & IHN-VL10A & -0.070 & 92 & IVL-UT150 & -0.072 \\
\hline 44 & IDN-BT16 & -0.074 & 93 & IBH-LS77 & 0.066 \\
\hline 45 & IDN-BT28 & -0.068 & 94 & IVL-HT71 & 0.074 \\
\hline 46 & IIBS-CD7-1 & 0.068 & 95 & IIGD-AB3-1 & -0.069 \\
\hline 47 & IIHN-AB23 & -0.071 & 96 & IILC-TG15 & 0.072 \\
\hline 48 & IINB-HN27-1 & 0.067 & 97 & IILC-TG31 & 0.073 \\
\hline 49 & IINK-PT10 & 0.075 & 98 & IIPLK-PL16 & -0.067 \\
\hline 99 & $\mathrm{IBH}-\mathrm{LS} 97^{-1}$ & 0.116 & 130 & IIMT-TH 25 & -0.148 \\
\hline 100 & IHN-HP7 & 0.082 & 131 & IIMT-TH7 & -0.148 \\
\hline 101 & IVL-HT121 & 0.082 & 132 & IIMT-TV11 & -0.141 \\
\hline 102 & IVL-HT325-1 & 0.098 & 133 & IIMX-DC34 & -0.148 \\
\hline 103 & ILS-HN7 & 0.078 & 134 & IINB-HN11-1 & 0.089 \\
\hline 104 & IBT-APD49-1 & 0.115 & 135 & IINB-HN24 & 0.102 \\
\hline 105 & IBH-TH59 & 0.097 & 136 & IINK-PT13 & 0.139 \\
\hline 106 & IVL-HT173-2 & 0.079 & 137 & IISC-PL29 & -0.132 \\
\hline 107 & IBH-TH70A & 0.098 & 138 & IITL-TV5-1 & -0.135 \\
\hline 108 & IHN-VL50 & 0.093 & 139 & IITL-TV7 & -0.129 \\
\hline 109 & IVL-HT123 & 0.087 & 140 & IITX-TL14 & -0.098 \\
\hline 110 & ILS-HN12 & 0.102 & 141 & IITX-TL20-1 & -0.129 \\
\hline 111 & IHP-MC4-1 & 0.108 & 142 & IIYB-CN24-1 & -0.135 \\
\hline 112 & IBH-LS80 & 0.110 & 143 & IICD-HN6 & 0.085 \\
\hline 113 & IDN-BT86 & 0.092 & 144 & IICD-VC4 & -0.133 \\
\hline 114 & IVL-HT320A & 0.090 & 145 & IICT-GD1 & 0.130 \\
\hline 115 & IHP-NB14A & -0.099 & 146 & IICT-GD4 & 0.142 \\
\hline 116 & ILS-HN22 & -0.094 & 147 & IIDK-TM29 & -0.101 \\
\hline 117 & IBH-HN16A & 0.096 & 148 & IIDK-TM45 & -0.136 \\
\hline 118 & IBH-HN48 & 0.146 & 149 & IIDL-PR31 & -0.145 \\
\hline 119 & IHN-HP2A & 0.136 & 150 & IIGD-APD2-1 & 0.090 \\
\hline 120 & IIAB-CL5 & -0.105 & 151 & IIHN-AB17 & -0.122 \\
\hline 121 & IIAS-KS10 & -0.138 & 152 & IIHN-AB20 & -0.090 \\
\hline 122 & IIAS-KS16 & -0.092 & 153 & IIHN-AB7 & -0.134 \\
\hline 123 & IIAS-KS22 & -0.132 & 154 & IIHN-MT15 & -0.102 \\
\hline 124 & IIAS-KS32 & -0.115 & 155 & IIBMT-DT12 & -0.112 \\
\hline 125 & IIBH-XL6 & 0.097 & 156 & IIBS-CD14 & 0.147 \\
\hline 126 & IHN-HP5 & 0.170 & 157 & IINK-PT6-1 & -0.165 \\
\hline 127 & IIBMT-DT14 & -0.158 & 158 & IIPLK-PL24 & -0.164 \\
\hline 128 & IIBMT-DT4 & 0.151 & 159 & IITT-TK29 & -0.153 \\
\hline 129 & IIBN-QT11-1 & 0.166 & 160 & IIAS-KS35 & -0.169 \\
\hline \multicolumn{6}{|c|}{ Differences with absolute values more $17.5 \mathrm{~cm}$ and not more $20 \mathrm{~cm}$} \\
\hline 161 & IBMT-APD30 & 0.182 & 163 & IINB-HN32-1 & 0.178 \\
\hline 162 & IVL-HT95 & 0.177 & 164 & IVL-HT73 & 0.195 \\
\hline
\end{tabular}

Experimental results show that in combination with the initial national quasigeoid model VIGAC2017, the national geodetic coordinates $B, L, H$ of geodetic point in VN2000 - 3D allow getting the national normal height $H^{\gamma}$ with the second order national normal height accuracy on the whole territory of Vietnam. In addition, the national geodetic coordinates $B, L, H$ of geodetic pointreceived from conversion of the global geodetic coordinates $\bar{B}, \bar{L}, \bar{H}$ of this geodetic point, obtained from the processing of GNSS data in ITRF according to the WGS84 global reference ellipsoidwith the using of satellite ephemeris with accuracy at level $\pm 2,5 \mathrm{~cm}$, to VN2000 - 3D. Experimental results will be presented in the next scientific article. It is necessary to pay attention to the 
factthat, at present, more $60 \%$ first and second orders benchmarks have been displaced on the terrain surface of Vietnam's territory. So with the purpose of development of the national spatial reference system in Vietnam, we must perfect the national first and second orders leveling networks in the near future.

\section{Discussions}

Abovepresented research results show that the initial national quasi-geoid model VIGAC2017 has the high accuracy and allows starting the construction of the initial spatial reference system, which guarantees to get the second order normal height by GNSS technology. That is seenas the first step to the perfectible construction of the national spatial reference system in the future.

However, with the accuracy at level $\pm 0,062 \mathrm{~m}$ the inital national quasi-geoid model VIGAC2917 does not satisfy the requirement of accuracy more than $\pm 0,040 \mathrm{~m}$ for the construction of the national spatial reference system by international regulation. An increase of accuracy of the final national quasi-geoid model will be accomplished by an increase of accuracy of the mixed quasigeoidmodel VIGAC2014 based on usage of detailed gravimetric data on territory of Vietnam.

The physical geodesy exists two methods for determination of quasi-geoid height by gravimetric data:

- The first method: Calculation of quasigeoif height by Stokes's integral.

- The second method: Correction of spherical harmonic coefficients of Earth's Gravitational Model (EGM) by approach of Colombo O.

The first method requires existence of gravimetric data around computational point with radius of near zone at $3^{\circ}$. This requirement can't be sastified for narrow and long country like Vietnam in the near futute. In addition, at present, there is no detailed gravimetric data in Lao and Campuchia. So the second method becomes more realistic and has been proposed to use (Ha minh Hoa, 2013c; Ha Minh Hoa, 2014a; Ha Minh Hoa, 2014b; Ha Minh Hoa et al. 2016a). Apart from that correction of spherical harmonic coefficients of EGM can be carried out based GNSS data on the first and second orders (Ha Minh Hoa, Nguyen Thi Thanh Huong, 2015a). Vietnam Institute of Geodesy and Cartography will carry out project "Detailed gravimetric measurement in mountainous regions of Vietnam" in the near future.

\section{Conclusions}

In the epoch of application of GNSS technology, the task of the construction of the national spatial reference system becomes the most important research content of high geodesy, that concentrates in itself the most important achievements in fields of the physical geodesy and geometrical geodesy. The key problem of the aforementioned task is the construction of the high accurate national quasi-geoid model. This scientific article presented results of the construction of the initial national quasi-geoid model with accuracy at the level of $\pm 6,2 \mathrm{~cm}$ and determination of the 07 coordinate transformation parameters from ITRF according to the WGS84 global reference ellipsoid to VN2000 - 3D according to the WGS84 national reference ellipsoid. The increase of accuracy of this national quasigeoid model to level more than $\pm 4,0 \mathrm{~cm}$ will be performed by the method of correction of spherical harmonic coefficients of Earth Gravitational Model EGM2008 based on detailed gravimetric data on the territory of Vietnam in the future.

\section{References}

Bursa M., Kenyon S., Kouba J., Sima Z., Vatrt V., Vitek V., Vojtiskova M., 2007. The geo-potential value W0 for specifying the relativistic atomic time scale and a global vertical reference system. Journal of Geodesy, 81(2), 103-110.

Gauss, C.F., 1828. Bestimmung des Breitenunterscchiedes zwischen den Sternwarten von Gottingen und Altona, Gottingen.

Ha Minh Hoa, Dang Hung Vo, Pham Hoang Lan, Nguyen Ngoc Lau, 2005. Research scientific base 
Ha Minh Hoa/Vietnam Journal of Earth Sciences 39 (2017)

for construction of different orders GPS networks in dynamic reference system. General report of the science - technological teme of the Ministry of Natural Resources and Environment in period 2002 - 2004, Hanoi.

Ha Minh Hoa et al., 2012. Research scientific base for perfection of the height system in connection with construction of national dynamic reference system. General report of the science - technological teme of the Ministry of Natural Resources and Environment. Hanoi.

Ha Minh Hoa, 2013a. Method of coordinate transformation bewwen coordinate systems. Science and Technique Publisher, Hanoi, 140p.

Ha Minh Hoa, 2013b. Estimating the geo-potential value $\mathrm{W}_{0}$ of the local geoid based on data from local and global normal heights of GPS/Leveling points in Vietnam. Geodesy and Cartography. Taylor \& Francis. UDK 528.21, doi:10.3846/20296991.2013.823705, 39 (3), 99-105.

Ha Minh Hoa, 2013c. Problems related to construction of national spatial reference system. Journal of Geodesy and Cartography, 18, 1-10. Vietnam Institute of Geodesy And Cartography.

Ha Minh Hoa, 2014a. Method of mathematical processing of national geodetic networks. Science and Technique Publisher, 244p, Hanoi.

Ha Minh Hoa, 2014b. Theory and practice of geodetic gravimetry. Science and Technique Publisher, Hanoi, 592p.

Ha Minh Hoa, Nguyen Thi Thanh Huong, 2015a. Research for perfection of method of mathematical processing of first, second orders leveling network in modern height system in Vietnam. General report of the science - technological teme of the Ministry of Natural Resources and Environment in period 20122015, Hanoi.

Ha Minh Hoa, Nguyen Tuan Anh, 2015b. Effective realization of correction of spherical harmonic coefficients of Earth gravitational model by mothod of Colombo O. L. Journal of Geodesy and Cartography, 25, 25-32. Vietnam Institute of Geodesy And Cartography.

Ha Minh Hoa, Nguyen Ba Thuy, Phan Trong Trinh et al., 2016a. Research for determination of normal surfaces of sea levels ("zero" depth surface, mean sea surface, highest sea surface) by methods of geodesy, hydrography and geology with serving construction of buildings and planning of coastline in tendency of climate changes". State techno scientific theme with code KC.09.19/11-15 in period of 2011-2015. Vietnam Ministry of Science and Technology, Ha Noi.

Ha Minh Hoa, 2016b. Research of height changes between Hon Dau local quasi-geoid and global quasi-geoid in the world scale. Journal of Geodesy and Cartography, 28, 1-7. Vietnam Institute of Geodesy And Cartography.

International Association of Geodesy (IAG), 1984. IAG Resolutions adopted at the XVIII General Assembly of the IUGG in Hamburg, August 1983. "The Geodesist's handbook”. Bulletin Geodetique, 58(3), p.321.

Listing, J.B., 1873. Ueber unsere jetzige Kenntuts der Gestalt und Grösse der Erde. Nachrichten von der Georg - Augusts Universi tät, Gröttingen, (3), 33-98.

Le Huy Minh, Tran Thi Lan, R. Fleury, C. Amory Mazaudier, Le Truong Thanh, Nguyen Chien Thang, Nguyen Ha Thanh, 2016. TEC variations and ionosphere disturbances during the magnetic storm in March 2015 observed from continuous GPS data in the Southeast Asia region. Vietnam Journal of Earth Sciences, 38(3), 287-305.

Luong Thanh Thach, 2016. Accuracy estimation of the initial national quasi-geoid model VIGAC2014 based on 75 second order benchmarks. Journal of Geodesy and Cartography, 25, 17-28. Vietnam Institute of Geodesy And Cartography.

Nguyen Tuan Anh, 2015. Detailed research of height of Hon Dau local geoid according to the global geoid in territory of Vietnam. Journal of Geodesy and Cartography, 25, 33-38. Vietnam Institute of Geodesy And Cartography.

Petit, G., Luzum, B., 2010. IERS Conventions, 2010. IERS Technical Note, 36, Verlag dés Bundesamts fur Kartographie und Geodasie. Frankfurt am Main 2010, 179p.

Scientific report. Construction of national coordinate system and reference system, 260p. General Department of Land Adminustration, Hanoi.

Vy Quoc Hai, Tran Quoc Cuong, Nguyen Viet Thuan, 2016. Crustal movement along the Red river fault from GNSS data. Vietnam Journal of Earth Sciences, 38(1), 14-21. 